American Journal of Applied Sciences 6 (1): 179-181, 2009

ISSN 1546-9239

(C) 2009 Science Publications

\title{
Association between Paternal Age at Birth Time and the Risk of Offspring Developing Schizophrenia
}

\author{
Victoria Omranifard and Ghorbanali Asadollahi \\ Department of Psychiatry, Isfahan Medical Sciences University, Iran
}

\begin{abstract}
Schizophrenia is the Leading psychotic disorder. It is a severe disease that involves social and interpersonal relationship of affected person and cause severe loss of function; that why many researchers wanted to know its etiologic factors. In this research, we wanted to reveal the relationship of paternal age to schizophrenia. This cross-sectional study was done on 240 patients of schizophrenia and 400 control person without any psychiatric and organic disorders. The average of paternal age at birth in schizophrenic group was $33.9 \pm 8.4$ years and that of control group was $32.7 \pm 8.3$ years $(\mathrm{P}=$ $0.04)$. In this study we concluded that the average of paternal age birth in schizophrenic patients was significantly higher than age of control group. This significancy show that schizophrenia, at least in part, is related to new mutations that originate in male germ cells. And this result can change our strategies to find the genetically etiology of schizophrenia and shows that father,s age no loss than mother's contributes variably to the risk of a suboptimal reproductive outcome.
\end{abstract}

Key words: Schizophrenia, paternal age at birth, maternal age

\section{INTRODUCTION}

Schizophrenia is a chronic devastating behavioural psychiatric disease whose history turn back to medical science ancientness. Less than $1 \%$ of world population attacked by schizophrenia. that have spread in every geographic region. Its prevalence and incidence rate is similar around the world but there is difference within both sexes and symptom appearances. Incidence rates were similar in rural and urban area, the prevalence of involvement is higher in the urban area and poor socioeconomic conditions. Since schizophrenia has premature outbreak, it has been associated with several great and long disorders, high hospitalization expenditure, medical care, rehabilitation and supportive service is needed. The cost of disease in united state is more than all kind of cancers ${ }^{[1]}$.

Although several factors have been mentioned in the etiology of schizophrenia. however, its etiology has been unknown. Several familiar twin and stepchild studies have established that genetic factors have role in the etiology of mentioned dysfunction. It is unknown the role of individual gene or group of gene cases disorder.

All family study has resulted in greater chance for schizophrenia in 1st degree relatives of patients in comparison with the community dwellings. Results from at least 19 great studies were confirmed, genetic factors have a great role in transmission of schizophrenia ${ }^{[1]}$. More than half a century ago, Book suggested that de novo mutations might cause genitival defect contribute responsibility of schizophrenia and also it is observed that increased paternal age is associated with increased mutations in his germ cells and increased paternal age is associated with de novo mutations ${ }^{[2]}$. Till now several studies have been done in case of investigation between increased paternal age and schizophrenia risks.

A study, was done by Colombia university researchers on 87907 cases, which showed that the prevalence in offspring whose fathers were aged 45-49 is twice in comparison with who are under 25 and in father who are up to 50 is triple than who are under 25 . This study showed that 26.6 percent of all schizophrenia is stronger relation to paternal age, while there is no relation for maternal transmission of the disease $^{[3]}$. Also a study was done in Sweden in the distribution of age 27 +years (754 cases), there was a relation between paternal age and schizophrenia in patient with no familiar history of schizophrenia and paternal age consider as a risk factor that shows mutation dysfunction in paternal cells ${ }^{[4]}$. Relation between schizophrenia pateints' father age and incidence of disease is not documented and clear in Iran. The aim of recent study is to determine this relationship.

Corresponding Author: Victoria Omranifard, Department of Psychiatry, Noor Hospital, Ostandari Street, Isfahan Medical Sciences University, Iran, Tel: 00989131156986 
Am. J. Applied Sci., 6(1): 179-181, 2009

\section{MATERIALS AND METHODS}

The case control study was designed and approved by research committee of Isfahan medical school. 240 cases of schizophrenia from two psychiatry centers: (1) A community psychiatry center (2) Special hospital for psychiatry was selected. For control individuals, 400 persons were collected randomly from the same district area in Isfahan city. Exclusion criterias for them were: substance abuse, chronic mental and cerebral disease like seizure et al, family history of any psychiatric disorders. They screen mentally by General Health Questionnaire 28-item and if necessary interviewed with psychiatrist to confirm.

The demographic data and variables were asked from patients care giver or guardian. All data were recorded into data collection form that prepared by authors.

The information was analyzed by SPSS-12 software. Pearson's Chi square test was chosen as the primary test for comparing difference between characteristics of patients. Student's t and MannWhitney tests compared the means between two groups. The analysis was done separately by genders and adjusted for age by Mantel-Haentzel test. P-value less than 0.05 were considered significantly.

\section{RESULTS AND DISCUSSION}

The demographic data were the same between two groups. Research finding show that the mean paternal age for offspring with schizophrenia was $33.9 \pm 8.4$ years (range 17-57), also finding show that the mean paternal age of controls for offspring was $32.7 \pm 8.3$ years (range 18-60).

Figure 1 shows different in two groups. Student's $t$ test showed significant differences between them $(\mathrm{P}=0.04)$. Table 1 showed the age of father in offspring birth day in two groups. Frequency of lower age was higher in case group. There was no linear association for father's age and schizophrenia occurance.

Our finding showed the mean paternal age for offspring in schizophrenia group was higher. It seems there is a relationship between paternal age and schizophrenia occurance and genetic factor has a roll. There were 5 studies that mentioned further mutation in paternal germ cells compared with maternal and in 24 studies, show increased paternal age is associated with increased disease due to genomic mutation rate. And in 8 studies that investigated on 10347 patients increasing psychological prevalence was related to increased

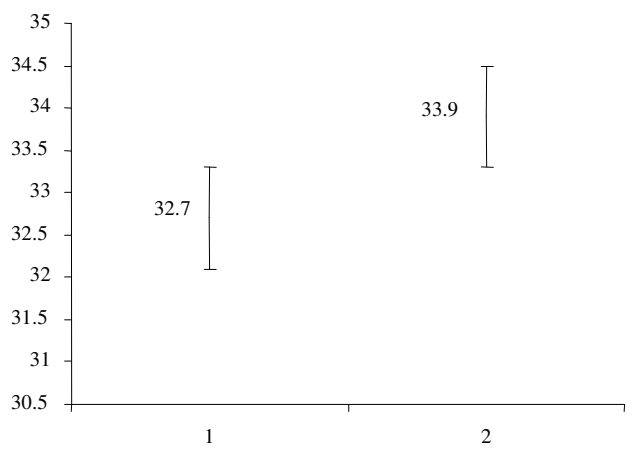

Fig. 1: The mean of paternal age in two study groups, $1=$ Normal, $2=$ Schizophrenia

Table 1: Frequency of age groups between cases and normal

\begin{tabular}{lll}
\hline Group & Control $(\%)$ & Case $(\%)$ \\
\hline$<25$ & 17.2 & 9.3 \\
$25-30$ & 17.5 & 20 \\
$31-35$ & 21.3 & 9 \\
$36-40$ & 17.9 & 12.1 \\
$41-45$ & 14.6 & 10.3 \\
$46-50$ & 7.9 & 34 \\
$50=<$ & 3.8 & 5.3 \\
\hline
\end{tabular}

paternal age and indicated paternal age as a health risk factor ${ }^{[5]}$. It has long been recognized that there is a relationship between maternal age and congenital disorders. Offspring of older fathers have an increased risk for maternal disorders related to DNA junctions during spermatogenesis.

Recent studies were noticed advancing paternal age was associated with an increasing incidence of schizophrenia ${ }^{[6]}$. In another study that was done by Rachka in 574 schizophrenic patients suggest that an increased risk of schizophrenia in older fathers ${ }^{[7]}$.

Then recognizing the association with paternal age is a step toward recognizing etiologic factors in schizophrenia. Many studies have declared the relationship between advanced paternal age and prevalence of schizophrenia in a family, in these surveys the linear correlation were found ${ }^{[7,8]}$, but we did not find any linear association in our finding.

At all, high paternal age at the time of birth of the offspring is a risk factor for adult schizophrenia. Alternative mechanisms for the mutagenesis effect are, contact to poison and nutrition defect, defect in DNA repair enzyme and so on. By finding result of this research it is seem that paternal age also as same as maternal age can cause unsuitable results. With the potential of making an impact on the course of schizophrenia by intervening early and preventing 
advanced paternal age at the time of birth would be a step in the right direction for schizophrenia prevention.

\section{ACKNOWLEDGMENT}

We have special thanks to Isfahan Behavioural Sciences Research Center to support this project. We appreciate to Dr Armin Barkatain and Mrs Zahra Ghazavi for data collection.

\section{REFERENCES}

1. Kaplan, H.I. and B.J. Sadock, 2004. Schizopherenia in: Kaplan, H.I. and B.J. Sadock, comprehensive Text book of Psychiatry. Philadelphia, Williams and Wilkins.

2. Book, J.A., 1953. Schizophrenia as a gene mutation. Acta Genetics, 4: 133-39.
3. Malaspina, D. and S. Harlap, 2001. Advancing paternal age and the risk of schizophrenia. Arch Gen. Psychiat, 58: 261-67.

4. Procopio, M., 2005. Paternal age and schizophrenia: one swallow does not make a summer. BMJ, 330 (7483): 147-48.

5. Attilasipos, M., 2004. Paternal age and schizophrenia apopulation based cohort study. BMJ, 329: 1070.

6. Ossama El-saadi, 2004. Paternal and maternal age as risk for psychosis; Findings from Denmark, Sweden and Australia. Schizophrenia Res., 67: 227-36.

7. Raschka, L.B., 2000. Paternal age and schizophren in dizygotic twins. Br. J. Psychiatr, 176: 400-01.

8. Bruce, L.A., 1979. Is psychosis related to father's age. Br. J. Psychiatr, 134: 169-77. 